\title{
Avaliação Morfométrica de Incisivos Centrais Superiores para Seleção de Implantes Imediatos em Maxila: Um Estudo Piloto
}

\author{
Morphometric Analysis of the Maxillary Central Incisors for Selection of Immediate Implants: A \\ Pilot Study
}

Evaluación Morfométrica de Incisivos Centrales Superiores para Selección de Implantes Maxilar

Inmediato: Un Estudio Piloto

Recebido: 31/01/2022 | Revisado: 05/02/2022 | Aceito: 06/02/2022 | Publicado: 12/02/2022

\author{
Marco Túllio Becheleni \\ ORCID: https://orcid.org/0000-0003-2576-3680 \\ Universidade Federal dos Vales do Jequitinhonha e Mucuri, Brasil \\ E-mail: marco@cirurgiabmf.com \\ Saulo Gabriel Moreira Falci \\ ORCID: https://orcid.org/0000-0001-9438-5199 \\ Universidade Federal dos Vales do Jequitinhonha e Mucuri, Brasil \\ E-mail: saulofalci@hotmail.com \\ Dimitri Ribas Fonseca \\ ORCID: https://orcid.org/0000-0002-4375-9274 \\ Universidade Federal dos Vales do Jequitinhonha e Mucuri, Brasil \\ E-mail: dimitri.ribas@gmail.com
}

\begin{abstract}
Resumo
Trata-se de análise morfométrica de incisivos centrais superiores com para auxílio no planejamento de implantes imediatos. A literatura revista não evidenciou um desenho de implante que permitisse condições ótimas de inserção imediata. Investigou-se um grupo compreendido por 25 incisivos centrais superiores extraídos (Grupo 1) e Grupo 2, corresponde a dois modelos de implantes, Alvim ${ }^{\circledR}$ Cone-Morse e Drive ${ }^{\circledR}$ Cone-Morse, indicados para reposição imediata de incisivos centrais superiores. No grupo 1, medidas diretas com auxílio de um paquímetro digital foram realizadas. Para o grupo 2, imagens bidimensionais dos implantes foram vetorizadas por software, viabilizando as mensurações e análises comparativas com o grupo 1. Após a análise estatística, a razão encontrada entre as médias dos diâmetros alveolares, numa localização apical de $-4 \mathrm{~mm}$ da junção cemento-esmalte ( $1 \mathrm{~mm}$ intraósseo) foi de 1,06. Desta forma, a radiografia periapical, pode sugerir valores relativamente seguros para previsão de dimensões visíveis apenas em tomografia computadorizada. Os achados revelaram que gaps estarão presentes para todas as plataformas de implantes comparadas. Após as mensurações, um modelo de implante compatível biomecanicamente com os supostos alvéolos poderia apresentar em sua configuração, uma plataforma medindo $6.5 \mathrm{~mm}$ de diâmetro, com alturas variadas, onde independente da altura, o diâmetro à $6 \mathrm{~mm}$ de sua plataforma seria de $4.5 \mathrm{~mm}$. Para a amostra tomada no estudo, este padrão de implante facilitaria um travamento primário dos implantes na região compreendida em 24 alvéolos (96\%). O estudo revelou uma grande discrepância entre supostos alvéolos e conformações de implantes investigados.
\end{abstract}

Palavras-chave: Cirurgia bucal; Implante dentário; Carga imediata em implante dentário; Alvéolo dental; Incisivo.

\begin{abstract}
This is a morphometric analysis of maxillary central incisors to aid in the planning of immediate implants. The literature reviewed did not show an implant design that would allow for optimal conditions for immediate insertion. A group comprising 25 extracted maxillary central incisors was investigated (Group 1) and Group 2, corresponding to two implant models, Alvim ${ }^{\circledR}$ Cone-Morse and Drive ${ }^{\circledR}$ Cone-Morse, indicated for immediate replacement of maxillary central incisors. In group 1, direct measurements with the aid of a digital caliper were performed. For group 2 , two-dimensional images of the implants were vectorized by software, enabling measurements and comparative analyzes with group 1. After the statistical analysis, the ratio found between the averages of the alveolar diameters, at an apical location of $-4 \mathrm{~mm}$ from the cemento- enamel (1mm intraosseous) was 1.06 . Thus, periapical radiography may suggest relatively safe values for predicting dimensions visible only on computed tomography. The findings revealed that gaps will be present for all implant platforms compared. After the measurements, an implant model biomechanically compatible with the supposed alveoli could present, in its configuration, a platform measuring $6.5 \mathrm{~mm}$ in diameter, with varying heights, where regardless of height, the diameter at $6 \mathrm{~mm}$ of its platform would be $4.5 \mathrm{~mm}$. For the sample taken in the study, this implant pattern would facilitate a primary locking of the implants in the
\end{abstract}


region comprised in 24 alveolus (96\%). The study revealed a large discrepancy between the supposed alveoli and conformations of investigated implants.

Keywords: Oral surgery; Dental implants; Immediate dental implant loading; Tooth socket; Incisor.

\section{Resumen}

Este es un análisis morfométrico de los incisivos centrales maxilares para ayudar en la planificación de implantes inmediatos. La literatura revisada no mostró un diseño de implante que permitiera condiciones óptimas para la inserción inmediata. Se investigó un grupo compuesto por 25 incisivos centrales maxilares extraídos (Grupo 1) y el Grupo 2, correspondiente a dos modelos de implantes, Alvim ${ }^{\circledR}$ Cone-Morse y Drive ${ }^{\circledR}$ Cone-Morse, indicados para el reemplazo inmediato de incisivos centrales maxilares. En el grupo 1 se realizaron mediciones directas con la ayuda de un pie de rey digital. Para el grupo 2, las imágenes bidimensionales de los implantes fueron vectorizadas por software, lo que permitió mediciones y análisis comparativos con el grupo 1. Después del análisis estadístico, la relación encontrada entre los promedios de los diámetros alveolares, en una ubicación apical de $-4 \mathrm{~mm}$ desde el cementoesmalte (1 mm intraóseo) fue de 1,06. Por lo tanto, la radiografía periapical puede sugerir valores relativamente seguros para predecir dimensiones visibles solo en tomografía computarizada. Los hallazgos revelaron que habrá lagunas en todas las plataformas de implantes comparadas. Tras las mediciones, un modelo de implante biomecánicamente compatible con los supuestos alvéolos podría presentar, en su configuración, una plataforma de 6,5 $\mathrm{mm}$ de diámetro, con alturas variables, donde independientemente de la altura, el diámetro a $6 \mathrm{~mm}$ de su plataforma sería de 4,5 mm. Para la muestra tomada en el estudio, este patrón de implantes facilitaría un bloqueo primario de los implantes en la región comprendida por 24 alvéolos (96\%). El estudio reveló una gran discrepancia entre los supuestos alvéolos y las conformaciones de los implantes investigados.

Palabras clave: Cirurgia bucal; Implantes dentales; Carga inmediata del implante dental; Alveolo dental; Incisivo.

\section{Introdução}

A inserção de implantes em alvéolos frescos tem sido um protocolo cirúrgico de sucesso (Esposito et al., 2007; Gelb, D. A., 1993; Gökçen-Röhlig et al., 2010). A preservação da arquitetura óssea alveolar (Grunder et al., 1999; Paolantonio et al., 2001), manutenção dos tecidos moles e diminuição de etapas cirúrgicas (Avvanzo et al., 2009; Covani et al., 2014; GökçenRöhlig et al., 2010) tem sido reportados como importantes vantagens desta conduta clínica.

A técnica de implante imediato requer a observância de requisitos biomecânicos como estabilidade mecânica inicial do implante e ótima arquitetura do alvéolo dentário. Além disso, o preenchimento do espaço existente entre osso e implante, bem como e a ausência de contato oclusal pós-operatório também têm influenciado no sucesso desta terapia (Gapski et al., 2003; Gillot, et al., 2012). Para implantes imediatos unitários em regiões estéticas, a manutenção longitudinal da arquitetura gengival parece estar também relacionada à geometria do implante (Stein et al., 2009) e ao posicionamento da plataforma de adaptação protética (Harel, et al., 2013; Lau et al., 2011).

Para a reposição de incisivos centrais, tem sido utilizados implantes cônicos ou cilíndricos, com diâmetros que variam de 2,5 a 5,0 mm e comprimento variando de 7 a $15 \mathrm{~mm}$ (Jofre, et al., 2012; Mozzati et al., 2012). A dimensão e conformação destes implantes objetiva o travamento cirúrgico supra-apical ao alvéolo, mas frequentemente proporciona um defeito de adaptação coronal entre o implante e a crista óssea adjacente tratada na literatura atual como gap.

Não foram encontradas evidências que conduzem à seleção de um desenho de implante que permita condições ótimas de inserção imediata pós-extração, minimizando o gap e as discrepâncias entre alvéolo e implante. Os implantes dentários têm apresentado poucas variações de conformação entre a indicação para o osso cicatrizado e alvéolos frescos (Mangano, et al., 2011; Marconcini, et al., 2013; Ormianer, et al., 2012).

Deste modo, o objetivo deste estudo piloto foi investigar a anatomia de incisivos centrais, mensurando dentes extraídos e comparando a desenhos de implantes, para melhor estabelecer a relação os implantes e a anatomia alveolar.

\section{Metodologia}

O presente estudo observacional investigou dois grupos amostrais, representados pelo Grupo 1 (G1), compreendendo incisivos centrais superiores extraídos (ICE) e pelo Grupo 2 (G2) que incluiu modelos de implantes com plataforma protética 
cone-morse (CM), indicados para reposição de incisivos. O estudo foi submetido ao comitê de ética em pesquisa pela Plataforma Brasil (CEP/CONEP, Ministério da Saúde, Brasil), e obteve-se parecer favorável (CAAE 19532213.2.0000.5108).

O universo de amostra inicial foi constituído por 58 ICE. Estes dentes foram oriundos de doações de um consultório particular, cujas exodontias foram realizadas mediante complicações de doenças periodontais, sem identificação dos doadores. Os critérios de inclusão dos ICE foram: 1) integridade anatômica do ápice; 2) junção cemento-esmalte identificável e 3) estrutura radicular que permitisse a mensuração dos pontos pré-definidos. Os dentes que não contemplavam os critérios de inclusão definidos foram excluídos. Assim, um total de 33 ICE foram excluídos do estudo e 25 ICE incluídos, constituindo o G1.

O G2 foi composto por dois modelos de implantes, Drive $\mathrm{CM}{ }^{\circledR}$ e Alvim $\mathrm{CM}$, (Neodent, Curitiba, Brasil), indicados para a técnica de implantes imediatos em região de incisivos centrais superiores.

Adotando metodologia semelhante de mensurações diretas das raízes por meio de paquímetro digital utilizada por Kim (2011), forma aferidas medidas com auxílio de um paquímetro digital (Mitutoyo, Santo Amaro, Brasil), adotadas para pontos distintos em cada ICE. As dimensões longitudinais radiculares foram mensuradas, entre o ápice e a junção cementoesmalte, sendo a mensuração mesial ACE1, distal ACE2, vestibular ACE3 e palatina ACE4 (Figura 1).

Figura 1: Esquema ilustrativo das mensurações longitudinais nos ICE.

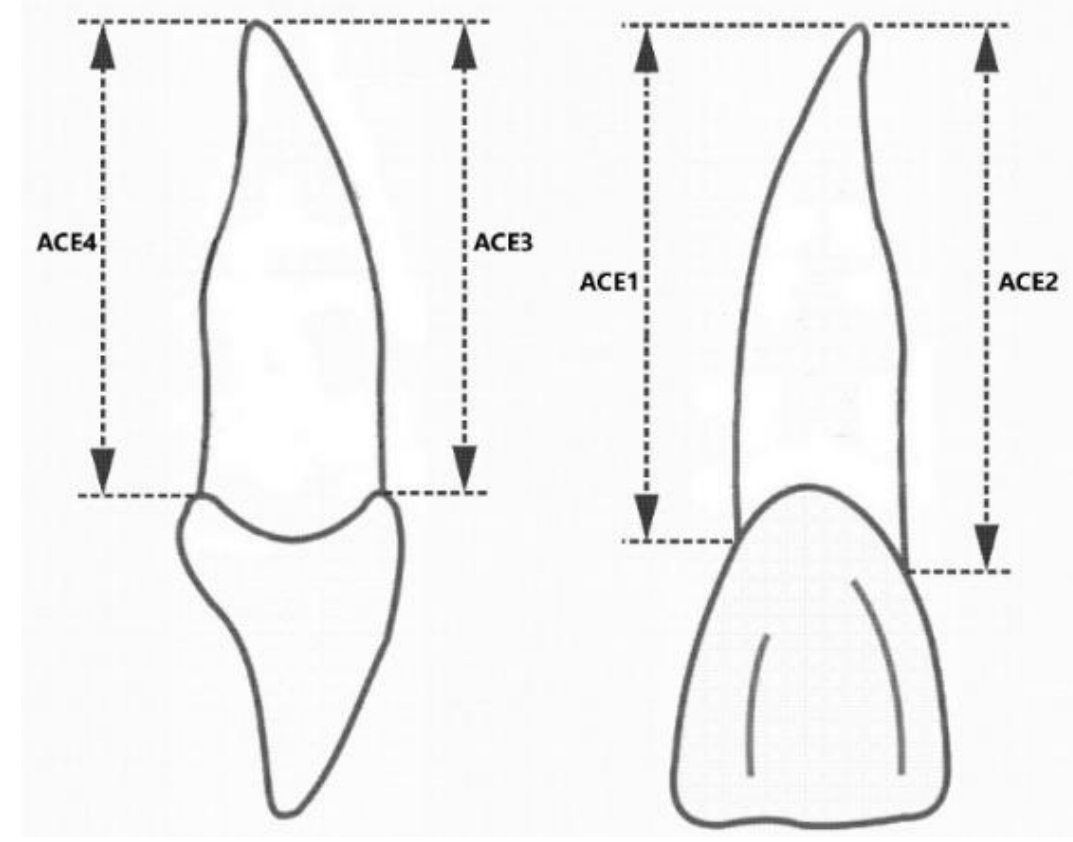

Fonte: Autores (2022).

Foram mensuradas ainda, de forma direta nos dentes extraídos, as dimensões lineares transversais das raízes ou diâmetros radiculares (Figura 2). Na suposta localização da crista óssea (DCO), revisada por Carranza et al. (2020) como 3mm apicais à JCE, foram tomadas a medida vestíbulo-palatina DCO3 e mésio-distal DCO8. Na região sugerida como ótima localização da plataforma protética do ICM, à 4 mm apicais em relação a JCE (Novaes et al. 2009), foram aferidas as medidas transversais DCO4 vestíbulo-palatina e DOC9 mésio-distal. Em uma porção radicular sugerida como início de uma maior conicidade apical, à 8 mm de distância apical em relação a JCE (Kim et al. 2011), foram mensurados DCO5 como diâmetro vestíbulo-lingual e DCO10 como diâmetro mésio-distal. Em uma distância apical de $10 \mathrm{~mm}$ em relação à JCE, área possível para um diâmetro alveolar menor que $4 \mathrm{~mm}$ (Kim et al. 2011), foram mensuradas as transversais DCO6 vestíbulo-palatina e DCO11 mésio-distal. 
Figura 2: Esquema ilustrativo das mensurações transversais nos ICE.

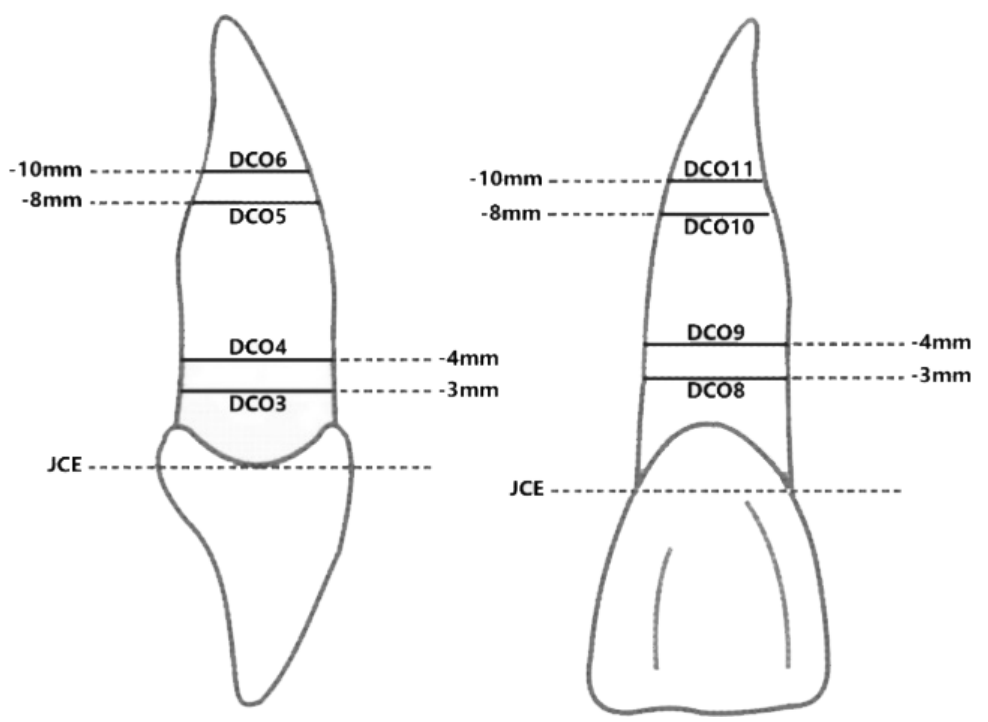

Fonte: Autores (2022).

A porção radicular de maior conicidade apical aparente (MCAA), onde a raiz passa a convergir de forma acentuada em direção apical foi avaliada. Mensurados dos diâmetros vestíbulo-palatinos e mésio-distais foram tomadas, utilizando-se o paquímetro digital (Mitutoyo, Santo Amaro, Brasil), bem como a altura desta região até a JCE (Figura 3).

Figura 3: Esquema ilustrativo das mensurações MCAA nos ICE.
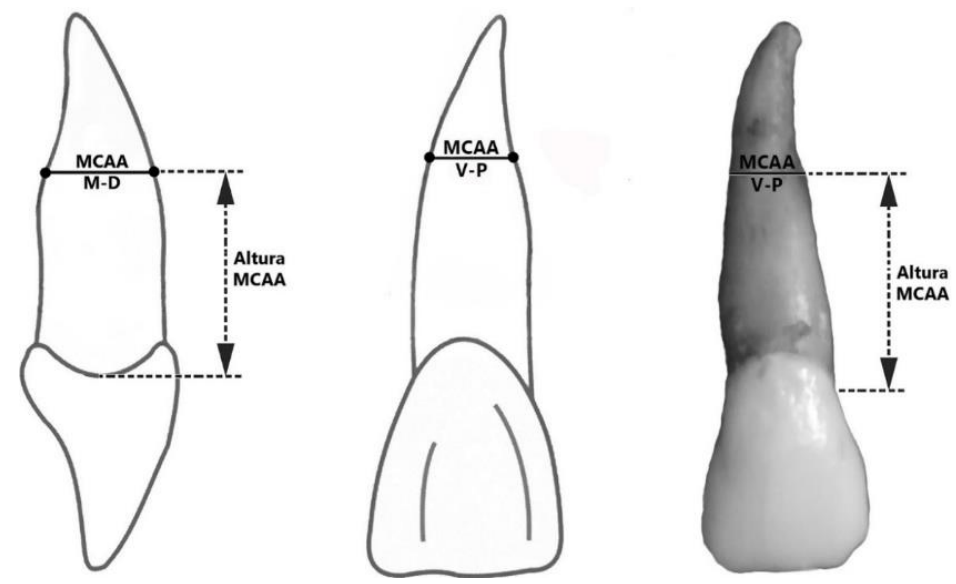

Fonte: Autores (2022).

A segunda etapa das análises do G1 contou com mensurações indiretas das raízes. Com auxílio de uma lupa com magnificação de 8x demarcou-se os incisivos com caneta hidrográfica nos pontos -3mm à JCE (DCO3 e DCO8), -4mm à JCE (DCO4 e DCO9) e na zona de maior conicidade apical aparente (MCAA V-P e MCAA M-D). Após demarcadas as áreas de interesse, os dentes foram incluídos individualmente em silicone odontológica densa (Zetalabor®, São Paulo, Brasil) dentro de um index cilíndrico gerando assim 25 corpos amostrais.

Após a presa do silicone, os cilindros foram separados dos dentes e seccionados transversalmente, na altura das marcações citadas e com auxílio de uma lâmina de bisturi n ${ }^{\circ} 11$. Formaram-se anéis de silicone (figura 4), que foram fotografados, juntamente com uma sonda periodontal milimetrada de Williams que serviu de parâmetro para calibração das fotografias. O alo negativo, contido em cada anel de silicone, é corresponde a uma secção transversal da raiz nos pontos 
selecionados (-3mm, -4mm apicais a JCE e no MCAA). O alo negativo, depois de manipulado por softwares de vetorização de imagens (CorelDraw X7, 2014, Ottawa, Canadá) e (ImageJ 1.48v, National Institutes of Health, USA), foram capazes de gerar mensurações quanto às áreas radiculares nos pontos (3mm, -4mm apicais a JCE e no MCAA).

Figura 4: Exemplo de um anel em silicone pesada, onde é possível observar um espaço negativo (negro), correspondente à raiz, e sonda milimetrada de Williams para auxiliar na calibração.

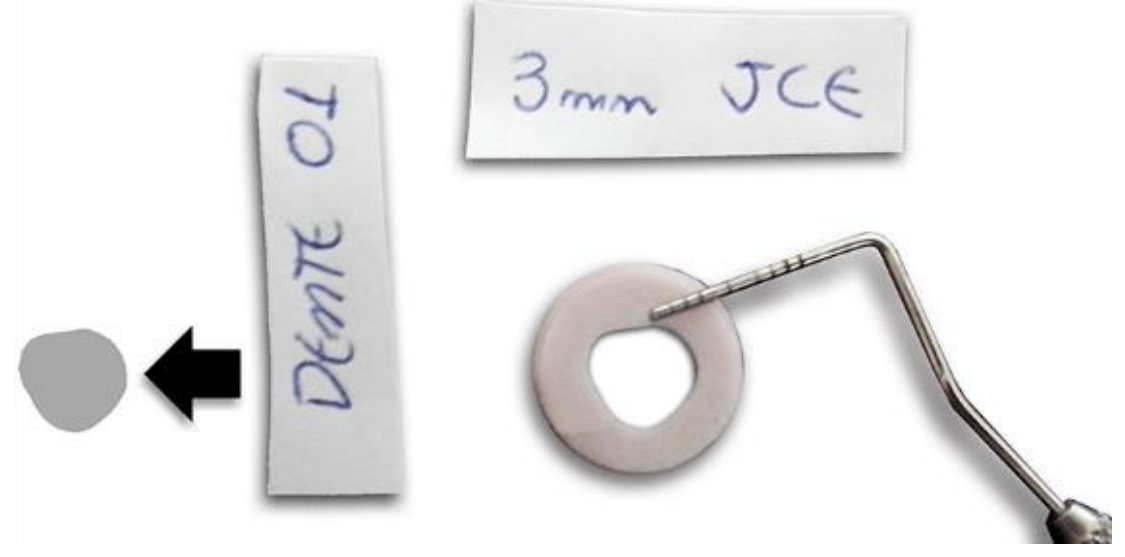

Fonte: Autores (2022).

O G2, composto pelos modelos de implantes Drive $\mathrm{CM} \circledast$ e Alvim $\mathrm{CM} \circledast$, apresentam um perfil cilíndrico em sua porção coronal e uma configuração cônica na região apical. Suas apresentações incluíram diâmetros de $3.5 \mathrm{~mm}$, $4.3 \mathrm{~mm}$ e $5 \mathrm{~mm}$ e alturas nas medidas de $10 \mathrm{~mm}, 11,5 \mathrm{~mm}, 13 \mathrm{~mm}$ e $16 \mathrm{~mm}$. As imagens bidimensionais dos implantes indicados pelo fabricante foram vetorizadas no software (CorelDraw X7, 2014, Ottawa, Canadá) para viabilizar as mensurações e análises comparativas com G1.

Os resultados das mensurações dos dois grupos foram tabulados em programa (Excel, Microsoft Office, Brasil). Durante a tabulação, foram criadas as projeções das dimensões alveolares, individualizada à cada raiz dentária. Para tanto, foram subtraídos 3mm nas dimensões longitudinais (Carranza et al. 2020), correspondentes a suposta localização da crista óssea (3mm à JCE), posteriormente foram acrescidos de $0,2 \mathrm{~mm}$, à cada face radicular, correspondendo ao espaço do ligamento periodontal, sugerido por Carranza et al. (2020) como 0,2 $\mathrm{mm}$ em média. Deste modo, os diâmetros aumentaram em 0,4mm e os comprimentos alveolares longitudinais foram $0,2 \mathrm{~mm}$ maiores que as raízes nesta região. A partir das áreas radiculares obtidas por meio dos os anéis de silicone, projeções das áreas alveolares foram realizadas. Para tanto, todo o perímetro do alo interno foi acrescido de 0,2mm, correspondente ao ligamento periodontal (Carranza et al. 2020). Os dados foram analisados estatisticamente no programa (SPSS Statistics v.22, IBM Corporation, EUA) para avaliação de possíveis associações.

\section{Resultados e Discussão}

As análises métricas longitudinais dos ICE, tomando como referencial as JCE proximais (ACE1 e ACE2), revelaram uma altura radicular média de 15,42mm. Partindo das JCE localizadas na face vestibular e palatina (ACE4 e ACE5), um comprimento radicular médio de $11,94 \mathrm{~mm}$ foi encontrado.

Na zona de maior conicidade apical aparente (MCAA), a altura foi aferida partindo-se das JCE proximais e revelou um comprimento médio de $8,51 \mathrm{~mm}$. 
A partir das mensurações radiculares, pôde-se fazer uma projeção dos supostos comprimentos alveolares. Para tanto, $0,2 \mathrm{~mm}$ foram acrescidos, correspondentes à espessura do ligamento periodontal na região apical e $3 \mathrm{~mm}$ subtraídos, referentes à distância da JCE até a suposta localização da crista óssea.

As projeções alveolares em relação às faces proximais (ACE1 e ACE2) apresentaram altura média de 12,62mm. O comprimento alveolar a partir das faces vestibular e palatina (ACE4 e ACE5), uma média de 9,04mm foi encontrada.

Para a altura de MCAA, apenas $3 \mathrm{~mm}$ foram removidos dos comprimentos radiculares correspondentes a distância deste ponto até a crista óssea alveolar, obteve-se assim a projeção da altura alveolar até o ponto MCAA. O comprimento médio encontrado foi $5,51 \mathrm{~mm}$.

A avaliação da média de comprimentos radiculares longitudinais revelou discrepância de 3,4mm, maior para as proximais quando comparadas às vestíbulo-palatinas. As medidas longitudinais dos ICE apresentaram grande variabilidade de comprimentos radiculares (Gráfico 1 e Tabela 1).

Gráfico 1: Projeções dos comprimentos alveolares médios (Gráfico Q-Q Normal).

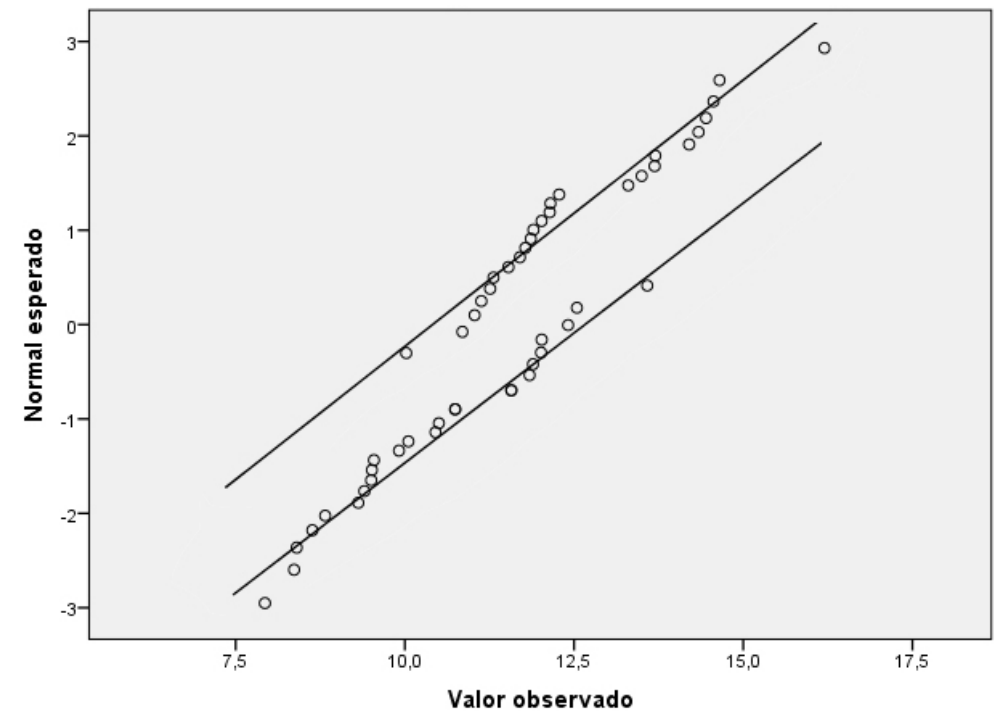

Superior revelando distribuição dos comprimentos alveolares médios em relação as faces M-D e inferior em relação as faces V-P. Fonte: Autores (2022).

Tabela 1: Analise descritiva dos comprimentos radiculares e projeções alveolares.

\begin{tabular}{lccccc}
\hline & média & sd. & min. & max. & intervalo \\
\cline { 2 - 6 } Comprimento radicular (ACE1 e ACE2) & 15,42 & 1,52 & 12,82 & 19 & 6,18 \\
Comprimento radicular (ACE4 e ACE5) & 11,94 & 1,71 & 8,98 & 15,77 & 6,79 \\
Comprimento radicular (MCAA) & 8,51 & 2,17 & 6,27 & 11,9 & 5,63 \\
Comprimento alveolar* (ACE1 e ACE2) & 12,62 & 1,52 & 10,02 & 16,20 & 6,18 \\
Comprimento alveolar* (ACE4 e ACE5) & 9,04 & 2,01 & 6,18 & 12,97 & 6,79 \\
Comprimento alveolar* (MCAA) & 5,51 & 1,27 & 3,27 & 8,9 & 5,63 \\
\hline
\end{tabular}

*Comprimentos alveolares foram estimados a partir dos dentes, desconsiderando o espaço do ligamento periodontal. Fonte: Autores (2022). 
A opção por um implante com $10 \mathrm{~mm}$ de comprimento, inserido a $1 \mathrm{~mm}$ dentro do alvéolo, define uma projeção de $11 \mathrm{~mm}$ apicais. Nesta comparação o Gráfico 2 apresenta regiões com zonas de corte em $11 \mathrm{~mm}, 12,5 \mathrm{~mm}, 14 \mathrm{~mm}$ e $17 \mathrm{~mm}$, proporcionais aos comprimentos de implante com 10mm, 11,5mm, 13mm e $16 \mathrm{~mm}$ respectivamente. Percebe-se que em 2 casos (8\%), o comprimento alveolar foi inferior ao implante de $10 \mathrm{~mm}$. Entre a faixa de comprimentos de implante de $10 \mathrm{~mm}$ e 11,5mm, 13 casos foram relatados (52\%). Na faixa que compreende entre o implante de $11,5 \mathrm{~mm}$ e o implante de $13 \mathrm{~mm}, 4$ casos foram encontrados (16\%), e 6 casos onde os comprimentos alveolares foram superiores ao implante de $13 \mathrm{~mm}$ (24\%). O ótimo travamento intraósseo de implantes imediatos, frequentemente tem sido reportado à inserção supra alveolar. Com este foco, a média de profundidade alveolar (12,62mm) sugere menor indicação dos implantes de $10 \mathrm{~mm}$ e $11,5 \mathrm{~mm}$.

Gráfico 2: Compatibilidade Comprimento de implantes x Profundidade alveolar*

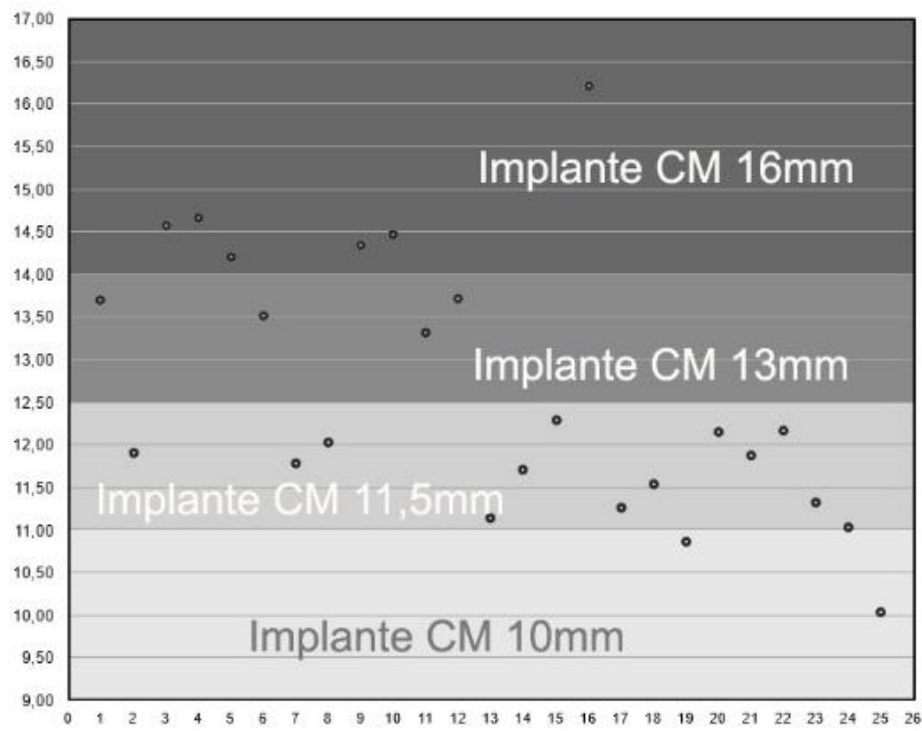

*Profundidade alveolar (mm) adaptada das dimensões radiculares. Fonte: Autores (2022).

Os valores encontrados nas mensurações transversais dos ICE, foram acrescidos de $0,4 \mathrm{~mm}$, correspondentes ao ligamento periodontal, representando assim projeções de diâmetros alveolares (Tabela 2). 
Tabela 2: Analise descritiva das projeções dos diâmetros alveolares*.

\begin{tabular}{|c|c|c|c|c|c|c|}
\hline Referência & Diâmetros & média & sd. & Min. & Max. & Intervalo \\
\hline \multirow{4}{*}{ JCE $-3 \mathrm{~mm}$} & $(\mathrm{~V}-\mathrm{P})$ & \multirow{2}{*}{6,55} & \multirow{2}{*}{0,24} & \multirow{2}{*}{6,15} & \multirow{2}{*}{6,95} & \multirow{2}{*}{0,8} \\
\hline & DCO3 & & & & & \\
\hline & (M-D) & \multirow{2}{*}{6,41} & \multirow{2}{*}{0,49} & \multirow{2}{*}{5,25} & \multirow{2}{*}{7,15} & \multirow{2}{*}{1,9} \\
\hline & DCO8 & & & & & \\
\hline \multirow{4}{*}{$\mathrm{JCE}-4 \mathrm{~mm}$} & $(\mathrm{~V}-\mathrm{P})$ & \multirow{2}{*}{6,21} & \multirow{2}{*}{0,37} & \multirow{2}{*}{5,44} & \multirow{2}{*}{7} & \multirow{2}{*}{1,56} \\
\hline & DCO4 & & & & & \\
\hline & (M-D) & \multirow{2}{*}{5,91} & \multirow{2}{*}{0,48} & \multirow{2}{*}{5,1} & \multirow{2}{*}{6,83} & \multirow{2}{*}{1,73} \\
\hline & DCO9 & & & & & \\
\hline \multirow{4}{*}{$\mathrm{JCE}-8 \mathrm{~mm}$} & $(\mathrm{~V}-\mathrm{P})$ & \multirow{2}{*}{5,44} & \multirow{2}{*}{0,45} & \multirow{2}{*}{4,4} & \multirow{2}{*}{6,4} & \multirow{2}{*}{2} \\
\hline & DCO5 & & & & & \\
\hline & (M-D) & \multirow{2}{*}{4,94} & \multirow{2}{*}{0,52} & \multirow{2}{*}{3,92} & \multirow{2}{*}{5,9} & \multirow{2}{*}{1,98} \\
\hline & DCO10 & & & & & \\
\hline \multirow{4}{*}{$\mathrm{JCE}-10 \mathrm{~mm}$} & (V-P) & \multirow{2}{*}{4,45} & 0.5 & 373 & 502 & 210 \\
\hline & DCO6 & & & & & \\
\hline & (M-D) & 300 & 052 & 312 & 517 & 205 \\
\hline & DCO11 & 5,99 & 0,32 & 5,12 & $J, 17$ & $2,0 \mathrm{~s}$ \\
\hline & (V-P) & 54 & 056 & 420 & 672 & 51 \\
\hline$M C \wedge A$ & MCAA & J,4 & 0,50 & 4,22 & 0,13 & 2,51 \\
\hline 19e & (M-D) & 40 & 066 & 376 & 624 & 50 \\
\hline & MCAA & 4,9 & 0,01 & $J, / 0$ & 0,34 & $2, J 0$ \\
\hline
\end{tabular}

*Diâmetros projetados sobre dimensões radiculares aferidas em mm. Fonte: Autores (2022).

Tomado como referencial a suposta localização da crista óssea alveolar (DCO3 e DCO8), revelou-se um diâmetro médio de $6,55 \mathrm{~mm}$ no sentido vestíbulo-palatino e $6,41 \mathrm{~mm}$ de diâmetro no sentido mésio-distal.

Na altura tomada para submersão da plataforma dos implantes (DCO4 e DCO9), a 1mm da crista óssea alveolar e $4 \mathrm{~mm}$ da JCE, as mensurações revelaram uma média de $6,21 \mathrm{~mm}$ de diâmetro no sentido vestíbulo-palatino e 5,91mm de diâmetro no sentido mésio-distal.

A razão entre as médias dos diâmetros alveolares em -4mm da JCE (1mm intraósseo) foi de 1,06. Desta forma, o planejamento incluindo radiografia periapical, que permite a mensuração do diâmetro mésio-distal do alvéolo, pode sugerir valores relativamente seguros para previsão da dimensão vestíbulo-palatina, visíveis apenas em exame tomográfico.

Na porção radicular sugerida como início de uma maior conicidade apical (DCO5 e DCO10), a 8 mm da JCE, as análises revelaram uma média de 5,44 mm de diâmetro no sentido vestíbulo-palatino e 4,94 mm de diâmetro no sentido mésiodistal.

Na área possível para um diâmetro alveolar menor que 4 mm (DCO6 e DCO11), a 10 mm da JCE, um diâmetro médio de 4,45 mm no sentido vestíbulo-palatino foi encontrado. No sentido mésio-distal, o diâmetro médio foi de 3,99 mm.

Na zona de maior conicidade apical aparente (MCAA), os diâmetros foram aferidos, revelando uma média de 5,4 mm no sentido vestíbulo-palatino e 4,9 mm no sentido mésio-distal. 
Os diâmetros médios para DCO4 e DCO9 ao serem comparados com as plataformas dos implantes Alvim ConeMorse ${ }^{\circledR}$ e Drive Cone-Morse ${ }^{\circledR}$ revelam sobre espaçamento (gap) vestíbulo-palatino de 2,71 mm e 2,41mm mésio-vestibular em relação a plataforma 3.5, um espaçamento vestíbulo-palatino de $1,91 \mathrm{~mm}$ e $1,61 \mathrm{~mm}$ mésio-vestibular em relação a plataforma 4.3 e um espaçamento vestíbulo-palatino de $1,21 \mathrm{~mm}$ e $0,91 \mathrm{~mm}$ mésio-vestibular em relação a plataforma 5.0.

Os achados revelam que gaps entre o alvéolo e a plataforma dos implantes estão presentes em todas as comparações realizadas.

Os diâmetros médios para DCO5 e DCO10, a 8mm da JCE e 5mm da crista óssea alveolar ao serem comparados com os diâmetros dos implantes Alvim Cone-Morse ${ }^{\circledR}$ e Drive Cone-Morse ${ }^{\circledR}$ (tabela 3), na altura equivalente a $4 \mathrm{~mm}$ de suas plataformas, revelam que um travamento primário às expensas das paredes vestibular e palatina pode ser conseguido através do implante Drive Cone-Morse ${ }^{\circledR}$ com plataforma 5.0 nos comprimentos $10 \mathrm{~mm}, 11,5 \mathrm{~mm}$ e $13 \mathrm{~mm}$ e no implante Alvim ConeMorse ${ }^{\circledR}$ de plataforma 5.0 nos comprimentos $13 \mathrm{~mm}$ e $16 \mathrm{~mm}$. Observa-se nos demais implantes de plataforma 5.0, uma discrepância positiva com baixos valores em ambos os modelos de implantes e em todas as alturas.

Tabela 3: Discrepância entre os diâmetros das projeções alveolares* e diâmetros dos implantes à 8mm apicais à JCE (DCO5 e DCO10).

\begin{tabular}{|c|c|c|c|c|c|}
\hline & comp. & plat. & Diâmetro & Discrepância Diâmetro V-P & Discrepância Diâmetro M-D \\
\hline & 10 & & 3,45 & 1,49 & 1,99 \\
\hline Alvim & 11,5 & & 3,45 & 1,49 & 1,99 \\
\hline \multirow[t]{3}{*}{$\mathrm{CM}$} & 13 & 3.5 & 3,45 & 1,49 & 1,99 \\
\hline & 16 & & 3,45 & 1,49 & 1,99 \\
\hline & 10 & & 3,47 & 1,47 & 1,97 \\
\hline Drive & 11,5 & & 3,48 & 1,46 & 1,96 \\
\hline \multirow[t]{3}{*}{$\mathrm{CM}$} & 13 & 3.5 & 3,42 & 1,52 & 2,02 \\
\hline & 16 & & 3,46 & 1,48 & 1,98 \\
\hline & 10 & & 4,25 & 0,69 & 1,19 \\
\hline Alvim & 11,5 & & 4,26 & 0,68 & 1,18 \\
\hline \multirow[t]{3}{*}{$\mathrm{CM}$} & 13 & 4.3 & 4,27 & 0,67 & 1,17 \\
\hline & 16 & & 4,26 & 0,68 & 1,18 \\
\hline & 10 & & 4,27 & 0,67 & 1,17 \\
\hline Drive & 11,5 & & 4,24 & 0,7 & 1,2 \\
\hline \multirow[t]{3}{*}{$\mathrm{CM}$} & 13 & 4.3 & 4,27 & 0,67 & 1,17 \\
\hline & 16 & & 4,25 & 0,69 & 1,19 \\
\hline & 10 & & 4,9 & 0,04 & 0,54 \\
\hline Alvim & 11,5 & & 4,93 & 0,01 & 0,51 \\
\hline \multirow[t]{3}{*}{$\mathrm{CM}$} & 13 & 5 & 4,94 & 0 & 0,5 \\
\hline & 16 & & 4,94 & 0 & 0,5 \\
\hline & 10 & & 4,95 & $-0,01$ & 0,49 \\
\hline Drive & 11,5 & & 4,97 & $-0,03$ & 0,47 \\
\hline \multirow[t]{2}{*}{$\mathrm{CM}$} & 13 & 5 & 5 & $-0,06$ & 0,44 \\
\hline & 16 & & 4,91 & 0,03 & 0,53 \\
\hline
\end{tabular}

*Considerando: Diâmetro médio V-P das projeções alveolares=4,94mm e diâmetro médio M-D=5,44mm. Fonte: Autores (2022). 
Os diâmetros médios para DCO6 e DCO11, a 10 mm da JCE e 7 mm da crista óssea alveolar, ao serem comparados com os diâmetros dos implantes Alvim Cone-Morse ${ }^{\circledR}$ e Drive Cone-Morse $\AA$ (Tabela 4), na altura equivalente a 6 mm de suas plataformas, revelam que um travamento primário pode ser conseguido através de ambos modelos com plataformas 4.3 e 5 em qualquer uma de seus comprimentos, com exceção do Alvim Cone-Morse® com 10mm de comprimento.

Tabela 4: Discrepância entre os diâmetros das projeções alveolares* e diâmetros dos implantes à 10mm da JCE (DCO6 e DCO11).

\begin{tabular}{|c|c|c|c|c|c|}
\hline & comp. & plat. & Diâmetro & Discrepância Diâmetro V-P & Discrepância Diâmetro M-D \\
\hline & 10 & & 3,12 & 1,33 & 0,87 \\
\hline Alvim & 11,5 & & 3,44 & 1,01 & 0,55 \\
\hline \multirow[t]{3}{*}{$\mathrm{CM}$} & 13 & 3.5 & 3,45 & 1 & 0,54 \\
\hline & 16 & & 3,45 & 1 & 0,54 \\
\hline & 10 & & 3,2 & 1,25 & 0,79 \\
\hline Drive & 11,5 & & 3,43 & 1,02 & 0,56 \\
\hline \multirow[t]{3}{*}{$\mathrm{CM}$} & 13 & 3.5 & 3,46 & 0,99 & 0,53 \\
\hline & 16 & & 3,49 & 0,96 & 0,5 \\
\hline & 10 & & 3,84 & 0,61 & 0,15 \\
\hline Alvim & 11,5 & & 4,25 & 0,2 & $-0,26$ \\
\hline \multirow[t]{3}{*}{$\mathrm{CM}$} & 13 & 4.3 & 4,23 & 0,22 & $-0,24$ \\
\hline & 16 & & 4,24 & 0,21 & $-0,25$ \\
\hline & 10 & & 4,24 & 0,21 & $-0,25$ \\
\hline Drive & 11,5 & & 4,04 & 0,41 & $-0,05$ \\
\hline \multirow[t]{3}{*}{$\mathrm{CM}$} & 13 & 4.3 & 4,18 & 0,27 & $-0,19$ \\
\hline & 16 & & 4,22 & 0,23 & $-0,23$ \\
\hline & 10 & & 4,46 & $-0,01$ & $-0,47$ \\
\hline Alvim & 11,5 & & 4,91 & $-0,46$ & $-0,92$ \\
\hline \multirow[t]{3}{*}{$\mathrm{CM}$} & 13 & 5 & 4,92 & $-0,47$ & $-0,93$ \\
\hline & 16 & & 4,91 & $-0,46$ & $-0,92$ \\
\hline & 10 & & 4,6 & $-0,15$ & $-0,61$ \\
\hline Drive & 11,5 & & 4,77 & $-0,32$ & $-0,78$ \\
\hline \multirow[t]{2}{*}{$\mathrm{CM}$} & 13 & 5 & 4,95 & $-0,5$ & $-0,96$ \\
\hline & 16 & & 4,85 & $-0,4$ & $-0,86$ \\
\hline
\end{tabular}

*Considerando: Diâmetro médio V-P das projeções alveolares=4,45mm e diâmetro médio M-D=3,99mm. Fonte: Autores (2022).

As projeções de diâmetros alveolares médios para MCAA, ao serem comparados com os diâmetros dos implantes Alvim Cone-Morse ${ }^{\circledR}$ e Drive Cone-Morse ${ }^{\circledR}$ (Tabela 5), na altura equivalente a 4,51mm de suas plataformas, equivalente a altura média do MCAA $(5,51 \mathrm{~mm})$ nas projeções alveolares subtraídas de $1 \mathrm{~mm}$, correspondente à região sugerida como ótima localização da plataforma protética do ICM, à 4mm apicais em relação a JCE e 1mm apical a crista óssea, revelam que um travamento primário pode ser obtido em ambos modelos de plataforma 5 em quaisquer alturas. 
Tabela 5: Discrepância entre os diâmetros das projeções alveolares* e diâmetros dos implantes na MCAA*.

\begin{tabular}{|c|c|c|c|c|c|}
\hline & comp. & plat. & Diâmetro & Discrepância Diâmetro V-P & Discrepância Diâmetro M-D \\
\hline \multirow{4}{*}{$\begin{array}{l}\text { Alvim } \\
\text { CM }\end{array}$} & 10 & \multirow{4}{*}{3.5} & 3,44 & 1,96 & 1,46 \\
\hline & 11,5 & & 3,44 & 1,96 & 1,46 \\
\hline & 13 & & 3,44 & 1,96 & 1,46 \\
\hline & 16 & & 3,45 & 1,95 & 1,45 \\
\hline \multirow{4}{*}{$\begin{array}{l}\text { Drive } \\
\mathrm{CM}\end{array}$} & 10 & \multirow{4}{*}{3.5} & 3,44 & 1,96 & 1,46 \\
\hline & 11,5 & & 3,45 & 1,95 & 1,45 \\
\hline & 13 & & 3,44 & 1,96 & 1,46 \\
\hline & 16 & & 3,47 & 1,93 & 1,43 \\
\hline \multirow{4}{*}{$\begin{array}{l}\text { Alvim } \\
\text { CM }\end{array}$} & 10 & \multirow{4}{*}{4.3} & 4,23 & 1,17 & 0,67 \\
\hline & 11,5 & & 4,25 & 1,15 & 0,65 \\
\hline & 13 & & 4,26 & 1,14 & 0,64 \\
\hline & 16 & & 4,25 & 1,15 & 0,65 \\
\hline \multirow{4}{*}{$\begin{array}{l}\text { Drive } \\
\text { CM }\end{array}$} & 10 & \multirow{4}{*}{4.3} & 4,27 & 1,13 & 0,63 \\
\hline & 11,5 & & 4,18 & 1,22 & 0,72 \\
\hline & 13 & & 4,28 & 1,12 & 0,62 \\
\hline & 16 & & 4,26 & 1,14 & 0,64 \\
\hline \multirow{4}{*}{$\begin{array}{l}\text { Alvim } \\
\text { CM }\end{array}$} & 10 & \multirow{4}{*}{5} & 4,9 & 0,5 & $\mathbf{0}$ \\
\hline & 11,5 & & 4,92 & 0,48 & $-0,02$ \\
\hline & 13 & & 4,94 & 0,46 & $-0,04$ \\
\hline & 16 & & 4,93 & 0,47 & $-0,03$ \\
\hline \multirow{4}{*}{$\begin{array}{l}\text { Drive } \\
\mathrm{CM}\end{array}$} & 10 & \multirow{4}{*}{5} & 4,9 & 0,5 & 0 \\
\hline & 11,5 & & 4,99 & 0,41 & $-0,09$ \\
\hline & 13 & & 4,98 & 0,42 & $-0,08$ \\
\hline & 16 & & 4,93 & 0,47 & $-0,03$ \\
\hline
\end{tabular}

*Considerando: Diâmetro médio V-P das projeções alveolares=5,4mm e diâmetro médio $\mathrm{M}-\mathrm{D}=4,9 \mathrm{~mm}$

*Diâmetros tomados nos implantes a partir da altura média de MCAA das projeções alveolares. Fonte: Autores (2022).

As áreas das secções transversais das projeções alveolares, em DCO3 e DCO8 (à 3mm da JCE, na altura da crista óssea alveolar), ao serem comparados com as áreas das plataformas dos implantes Alvim Cone-Morse® e Drive Cone-Morse ${ }^{\circledR}$ (Tabela 6), revelaram que as áreas alveolares foram maiores em $100 \%$ dos casos. Os achados revelam que gaps entre o alvéolo e a plataforma dos implantes estariam presentes em todas as comparações, caso o implante fosse justaposto a crista óssea alveolar. 
Tabela 6: Discrepâncias* entre as áreas das secções transversais das projeções alveolares individuais e das plataformas dos implantes Alvim Cone-Morse ${ }^{\circledR}$ e Drive Cone-Morse $®$, na altura da crista óssea alveolar.

\begin{tabular}{|c|c|c|c|c|c|c|c|}
\hline \multirow[b]{2}{*}{ Alvéolo } & \multicolumn{3}{|c|}{ Discrepâncias } & \multirow[b]{2}{*}{ Alvéolo } & \multicolumn{3}{|c|}{ Discrepâncias } \\
\hline & 3.5 & 4.3 & 5 & & 3.5 & 4.3 & 5 \\
\hline 1 & 24,40 & 19,51 & 14,39 & 16 & 19,81 & 14,92 & 9,80 \\
\hline 2 & 16,92 & 12,03 & 6,91 & 17 & 23,07 & 18,18 & 13,06 \\
\hline 3 & 15,22 & 10,32 & 5,20 & 18 & 24,33 & 19,44 & 14,32 \\
\hline 4 & 18,39 & 13,49 & 8,37 & 19 & 21,41 & 16,52 & 11,40 \\
\hline 5 & 15,98 & 11,09 & 5,97 & 20 & 10,45 & 5,56 & 0,44 \\
\hline 6 & 16,25 & 11,35 & 6,23 & 21 & 15,97 & 11,08 & 5,96 \\
\hline 7 & 22,00 & 17,10 & 11,98 & 22 & 13,24 & 8,35 & 3,23 \\
\hline 8 & 23,56 & 18,67 & 13,55 & 23 & 17,38 & 12,49 & 7,37 \\
\hline 9 & 14,83 & 9,94 & 4,82 & 24 & 15,09 & 10,20 & 5,08 \\
\hline 10 & 15,77 & 10,88 & 5,76 & 25 & 22,08 & 17,19 & 12,07 \\
\hline 11 & 19,82 & 14,92 & 9,80 & & & & \\
\hline 12 & 23,07 & 18,18 & 13,06 & & & & \\
\hline 13 & 24,34 & 19,44 & 14,32 & & & & \\
\hline 14 & 21,41 & 16,52 & 11,40 & & & & \\
\hline 15 & 10,458 & 5,56 & 0,44 & & & & \\
\hline
\end{tabular}

* Valores em mm³. Fonte: Autores (2022).

Nas áreas das secções transversais das projeções alveolares, em DCO4 e DCO9 (à 4mm da JCE e à 1mm altura da crista óssea alveolar), ao serem comparadas às áreas das plataformas dos implantes (Tabela 7), os achados revelaram que em 24 alvéolos (92\%), as áreas alveolares foram maiores que as áreas das plataformas dos implantes. 
Tabela 7: Discrepâncias* entre as áreas das secções transversais das projeções alveolares individuais e das plataformas dos implantes Alvim Cone-Morse ${ }^{\circledR}$ e Drive Cone-Morse ${ }^{\circledR}$, à $1 \mathrm{~mm}$ da crista óssea alveolar.

\begin{tabular}{|c|c|c|c|c|c|c|c|}
\hline \multirow[b]{2}{*}{ Alvéolo } & \multicolumn{3}{|c|}{ Discrepâncias } & \multirow[b]{2}{*}{ Alvéolo } & \multicolumn{3}{|c|}{ Discrepâncias } \\
\hline & 3.5 & 4.3 & 5 & & 3.5 & 4.3 & 5 \\
\hline 1 & 16,70 & 11,81 & 6,69 & 16 & 14,97 & 10,08 & 4,96 \\
\hline 2 & 13,32 & 8,43 & 3,31 & 17 & 18,63 & 13,74 & 8,62 \\
\hline 3 & 12,55 & 7,66 & 2,54 & 18 & 18,16 & 13,27 & 8,15 \\
\hline 4 & 15,85 & 10,96 & 5,84 & 19 & 18,41 & 13,52 & 8,40 \\
\hline 5 & 12,46 & 7,57 & 2,45 & 20 & 11,82 & 6,93 & 1,81 \\
\hline 6 & 12,75 & 7,86 & 2,74 & 21 & 12,71 & 7,82 & 2,70 \\
\hline 7 & 20,93 & 16,04 & 10,92 & 22 & 2,86 & $-2,03$ & $-7,15$ \\
\hline 8 & 17,77 & 12,88 & 7,76 & 23 & 14,71 & 9,82 & 4,70 \\
\hline 9 & 14,95 & 10,06 & 4,94 & 24 & 12,96 & 8,07 & 2,95 \\
\hline 10 & 19,69 & 14,80 & 9,68 & 25 & 17,05 & 12,16 & 7,04 \\
\hline 11 & 18,63 & 13,74 & 8,62 & & & & \\
\hline 12 & 18,16 & 13,27 & 8,15 & & & & \\
\hline 13 & 18,41 & 13,52 & 8,40 & & & & \\
\hline 14 & 11,82 & 6,93 & 1,81 & & & & \\
\hline 15 & 18,63 & 13,74 & 8,62 & & & & \\
\hline
\end{tabular}

* Valores em $\mathrm{mm}^{3}$. Fonte: Autores (2022).

As médias das áreas das secções transversais das projeções alveolares, em MCAA, ao serem comparados com as áreas das secções dos implantes Alvim Cone-Morse ${ }^{\circledR}$ e Drive Cone-Morse ${ }^{\circledR}$ (Tabela 8), também em MCAA, revelaram que apenas o implante Alvim Cone-Morse $\AA$ de plataforma 5.0 e altura $16 \mathrm{~mm}$ apresentou discrepância negativa, representando um possível travamento primário com este modelo no ponto MCAA.

Tabela 8: Discrepâncias, em milímetros, entre as áreas médias MCAA das secções transversais dos alvéolos e das médias das áreas dos implantes em MCAA.

\begin{tabular}{|c|c|c|c|c|}
\hline \multicolumn{2}{|r|}{ comp. } & \multicolumn{3}{|c|}{ discrepâncias } \\
\hline \multirow{5}{*}{$\begin{array}{c}\text { Alvim } \\
\text { CM }\end{array}$} & plataformas: & 3.5 & 4.3 & 5 \\
\hline & 10 & 13,78 & 11,77 & 9,76 \\
\hline & 11,5 & 11,78 & 8,94 & 5,78 \\
\hline & 13 & 10,20 & 6,59 & 2,41 \\
\hline & 16 & 8,60 & 3,98 & $-0,26$ \\
\hline \multirow{4}{*}{$\begin{array}{c}\text { Drive } \\
\mathrm{CM}\end{array}$} & 10 & 11,96 & 8,41 & 6,77 \\
\hline & 11,5 & 10,87 & 8,12 & 5,09 \\
\hline & 13 & 9,39 & 6,85 & 3,70 \\
\hline & 16 & 8,46 & 5,14 & 2,72 \\
\hline
\end{tabular}

Fonte: Autores (2022).

As comparações de áreas entre as projeções alveolares e áreas dos implantes demonstram que apenas o implante Alvim Cone-Morse ${ }^{\circledR}$ de plataforma 5.0 e altura 16mm apresentou discrepância negativa. Os resultados apresentados conflitam 
quando comparados as discrepâncias de áreas e diâmetros. Ao conflito, atribuímos o fato de que os alvéolos apresentam secções transversais irregulares e não cilíndricos, porém, as dimensões dos diâmetros configuram um parâmetro mais simples e seguro para sugerir as possibilidades de travamentos implantares iniciais.

\section{Conclusão}

A razão encontrada entre as médias dos diâmetros alveolares em -4mm da JCE (1mm intraósseo) foi de 1,06. Desta forma, o planejamento incluindo radiografia periapical, que permite a mensuração do diâmetro mésio-distal do alvéolo, pode sugerir valores relativamente seguros para previsão da dimensão vestíbulo-palatina, visíveis apenas em exame tomográfico.

Os achados revelam que gaps entre o alvéolo e a plataforma dos implantes estarão presentes em todas as plataformas disponíveis.

Os implantes Alvim Cone-Morse $®$ e Drive Cone-Morse $®$, na altura equivalente a $6 \mathrm{~mm}$ de suas plataformas, revelam que um travamento primário parece ser possível através de ambos modelos com plataformas 4.3 e 5 em qualquer um de seus comprimentos, com exceção do Alvim Cone-Morse ${ }^{\circledR}$ com 10mm de comprimento.

O estudo piloto revelou discrepâncias entre supostos alvéolos de incisivos centrais e configurações de implantes indicados para sua reposição.

Como sugestão para estudos clínicos futuros, uma configuração de implante que contemplasse geometricamente as supostas medidas alveolares aferidas, deveria apresentar $6.5 \mathrm{~mm}$ de diâmetro, com redução para $4.5 \mathrm{~mm}$ à uma altura apical de $6 \mathrm{~mm}$ de sua plataforma. Para a amostra tomada no estudo, este padrão de implante facilitaria um travamento primário em 24 alvéolos (96\%), o que poderia ser testado em novos estudos experimentais.

\section{Referências}

Avvanzo, P., Ciavarella, D., Avvanzo, A., Giannone, N., Carella, M., \& Lo Muzio, L. (2009). Immediate placement and temporization of implants: three- to five-year retrospective results. The Journal of Oral Implantology, 35(3), 136-142. https://doi.org/10.1563/1548-1336-35.3.136

Carranza, F. A., Newmam, M. G., Takei, H. H., Klokkevold, P. R. Periodontia clínica. Guanabara Koogan, $13^{\mathrm{a}}$ edição, 2020

Covani, U., Canullo, L., Toti, P., Alfonsi, F., \& Barone, A. (2014). Tissue stability of implants placed in fresh extraction sockets: a 5-year prospective singlecohort study. Journal of Periodontology, 85(9), e323-332. https://doi.org/10.1902/jop.2014.140175

Esposito, M., Grusovin, M. G., Willings, M., Coulthard, P., \& Worthington, H. V. (2007). The effectiveness of immediate, early, and conventional loading of dental implants: a Cochrane systematic review of randomized controlled clinical trials. The International Journal of Oral \& Maxillofacial Implants, 22(6), 893-904. http://www.ncbi.nlm.nih.gov/pubmed/18271370

Gapski, R., Wang, H.-L., Mascarenhas, P., \& Lang, N. P. (2003). Critical review of immediate implant loading. Clinical Oral Implants Research, 14(5), 515527. https://doi.org/10.1034/j.1600-0501.2003.00950.x

Gelb, D. A. (1993). Immediate implant surgery: three-year retrospective evaluation of 50 consecutive cases. The International Journal of Oral \& Maxillofacial Implants, 8(4), 388-399. http://www.ncbi.nlm.nih.gov/pubmed/8270307

Gillot, L., Cannas, B., Buti, J., \& Noharet, R. (2012). A retrospective cohort study of 113 patients rehabilitated with immediately loaded maxillary cross-arch fixed dental prostheses in combination with immediate implant placement. European Journal of Oral Implantology, 5(1), 71-79. http://www.ncbi.nlm.nih.gov/pubmed/22518381

Gökçen-Röhlig, B., Meriç, U., \& Keskin, H. (2010). Clinical and radiographic outcomes of implants immediately placed in fresh extraction sockets. Oral Surgery, Oral Medicine, Oral Pathology, Oral Radiology, and Endodontology, 109(4), e1-e7. https://doi.org/10.1016/j.tripleo.2009.11.030

Grunder, U., Polizzi, G., Goené, R., Hatano, N., Henry, P., Jackson, W. J., Kawamura, K., Köhler, S., Renouard, F., Rosenberg, R., Triplett, G., Werbitt, M., \& Lithner, B. (1999). A 3-year prospective multicenter follow-up report on the immediate and delayed-immediate placement of implants. The International Journal of Oral \& Maxillofacial Implants, 14(2), 210-216. http://www.ncbi.nlm.nih.gov/pubmed/10212537

Harel, N., Moses, O., Palti, A., \& Ormianer, Z. (2013). Long-term results of implants immediately placed into extraction sockets grafted with $\beta$-tricalcium phosphate: a retrospective study. Journal of Oral and Maxillofacial Surgery: Official Journal of the American Association of Oral and Maxillofacial Surgeons, 71(2), e63-68. https://doi.org/10.1016/j.joms.2012.09.022

Jofre, J., Valenzuela, D., Quintana, P., \& Asenjo-Lobos, C. (2012). Protocol for immediate implant replacement of infected teeth. Implant Dentistry, 21(4), 287-294. https://doi.org/10.1097/ID.0b013e31825cbcf8 
Research, Society and Development, v. 11, n. 3, e7011326353, 2022

(CC BY 4.0) | ISSN 2525-3409 | DOI: http://dx.doi.org/10.33448/rsd-v11i3.26353

Kim, J.-H., Lee, J.-G., Han, D.-H., \& Kim, H.-J. (2011). Morphometric analysis of the anterior region of the maxillary bone for immediate implant placement using micro-CT. Clinical Anatomy (New York, N.Y.), 24(4), 462-468. https://doi.org/10.1002/ca.21101

Lau, S. L., Chow, J., Li, W., \& Chow, L. K. (2011). Classification of maxillary central incisors-implications for immediate implant in the esthetic zone. Journal of Oral and Maxillofacial Surgery: Official Journal of the American Association of Oral and Maxillofacial Surgeons, 69(1), 142-153. https://doi.org/10.1016/j.joms.2010.07.074

Mangano, F., Mangano, C., Ricci, M., Sammons, R. L., Shibli, J. A., \& Piattelli, A. (2011). Single-tooth Morse taper connection implants placed in fresh extraction sockets of the anterior maxilla: an aesthetic evaluation. Clinical Oral Implants Research, 23(11), 1302-1307. https://doi.org/10.1111/j.16000501.2011.02307.x

Marconcini, S., Barone, A., Gelpi, F., Briguglio, F., \& Covani, U. (2013). Immediate Implant Placement in Infected Sites: A Case Series. Journal of Periodontology, 84(2), 196-202. https://doi.org/10.1902/jop.2012.110279

Mozzati, M., Arata, V., Gallesio, G., Mussano, F., \& Carossa, S. (2012). Immediate postextraction implant placement with immediate loading for maxillary full-arch rehabilitation. The Journal of the American Dental Association, 143(2), 124-133. https://doi.org/10.14219/jada.archive.2012.0122

Novaes, A. B., Barros, R. R. M., Muglia, V. A., \& Borges, G. J. (2009). Influence of interimplant distances and placement depth on papilla formation and crestal resorption: a clinical and radiographic study in dogs. The Journal of Oral Implantology, 35(1), 18-27. https://doi.org/10.1563/1548-1336-35.1.18

Ormianer, Z., Piek, D., Livne, S., Lavi, D., Zafrir, G., Palti, A., \& Harel, N. (2012). Retrospective Clinical Evaluation of Tapered Implants. Implant Dentistry, 21(4), 350-356. https://doi.org/10.1097/id.0b013e31825feb16

Paolantonio, M., Dolci, M., Scarano, A., d'Archivio, D., di Placido, G., Tumini, V., \& Piattelli, A. (2001). Immediate implantation in fresh extraction sockets. A controlled clinical and histological study in man. Journal of Periodontology, 72(11), 1560-1571. https://doi.org/10.1902/jop.2001.72.11.1560

Stein, A. E., McGlmphy, E. A., Johnston, W. M., \& Larsen, P. E. (2009). Effects of implant design and surface roughness on crestal bone and soft tissue levels in the esthetic zone. The International Journal of Oral \& Maxillofacial Implants, 24(5), 910-919. http://www.ncbi.nlm.nih.gov/pubmed/19865632 